\title{
Resultados de la esofaguectomía en 53 pacientes con adenocarcinoma del esófago y Barrett extenso*
}

\author{
Drs. ATTILA CSENDES J. ${ }^{1}$, ITALO BRAGHETTO M. ${ }^{1}$, GONZALO CARDEMIL H. ${ }^{1}$, \\ E.U. SOLANGE CORTÉS L. ${ }^{1}$, Dr. MAHER MUSLEH K. ${ }^{1}$ \\ 1 Departamento de Cirugía, Hospital Clínico de la Universidad de Chile, Santiago, Chile.
}

\begin{abstract}
Results of esophagectomy among patients with esophageal cancer and Barrett esophagus

Background: The incidence of esophageal carcinoma has increased notoriously worldwide. Aim: To assess clinical features, immediate surgical results and long term survival of patients with esophageal carcinoma and Barrett esophagus. Material and Methods: Retrospective review of medical records of all patients operated for esophageal carcinoma, between 1996 y 2011. Results: The records of 53 patients aged $58 \pm 9$ years (45 males) were analyzed. The number of operated patients increased from 7 in the period 1968-1983 to 31 in the period 2004-2011. Peritoneal metastases were found in two patients, precluding a resection. Video assisted trans-hiatal approach was the most commonly used technique followed by minimally invasive thoracoscopic and laparoscopic surgery. Transit reconstruction was performed ascending the stomach to the neck in $90 \%$ of patients. The average length of Barrett esophagus was $7.4 \mathrm{~cm}$ and the mean number of excised lymph nodes was 19 . Ten patients had an incipient cancer and their five years survival was $80 \%$. The survival of those with tumors involving the muscular layer and those with transmural cancer was 25 and $5 \%$, respectively. Conclusions: There is an increase in the incidence of esophageal cancer in the last 10 years. The survival after surgery is highly dependent on the invasiveness of the tumor.
\end{abstract}

Key words: Esophageal cancer, Barrett esophagus, surgery.

\section{Resumen}

Introducción: El adenocarcinoma esofágico es el tumor que ha experimentado el mayor aumento en su incidencia a nivel mundial. Objetivo: Determinar las características clínicas, los resultados inmediatos de la cirugía empleada y la sobrevida a largo plazo. Material y Método: Es un estudio retrospectivo y descriptivo incluyendo a todos los pacientes con adenocarcinoma de esófago entre 1996 y 2011. Se analizó la morbimortalidad operatoria y la sobrevida a 5 años. Resultados: Se aprecia un aumento de 10 veces su frecuencia comparada con 40 años atrás. Hay un claro predominio del sexo masculino de 6:1. Dos pacientes no se pu-

*Recibido el 8 de octubre de 2012 y aceptado para publicación el 10 de noviembre de 2012.

Los autores no refieren conflictos de interés.

Correspondencia: Dr. Attila Csendes J. Santos Dumont 999, Santiago, Chile. acsendes@redclinicauchile.cl 
dieron resecar por la presencia de metástasis peritoneales. El abordaje principal fue una técnica transhiatal video asistida, seguida de abordaje mini-invasivo toracoscópico y laparoscópico. La reconstitución del tránsito gastrointestinal fue en un $90 \%$ con estómago ascendido hasta el cuello. La morbilidad más frecuente fue la fístula cervical y la mortalidad de 5,7\%. El largo promedio del esófago de Barrett fue de 7,4 cm y el promedio de linfonodos resecados fue de 19 . Hubo 10 pacientes con adenocarcinoma incipiente, cuya sobrevida a 5 años fue de $80 \%$, muy superior al cáncer muscular con sobrevida $25 \%$ y al cáncer transmural con sobrevida de $5 \%(\mathrm{p}<0,01)$. Conclusiones: Se aprecia un enorme aumento del adenocarcinoma esofágico en los últimos 10 años. El abordaje actual que parece ser muy promisorio es la técnica mini-invasiva. La sobrevida a 5 años depende de la profundidad de la infiltración tumoral.

Palabras clave: Adenocarcinoma de esófago, Barrett, cirugía.

\section{Introducción}

El esófago de Barrett o síndrome de Barrett se define como una condición en la que el epitelio escamoso del esófago distal es reemplazado por epitelio columnar, que se aprecia claramente durante el estudio endoscópico y el cual contiene una mucosa anormal con metaplasia intestinal incompleta y células caliciformes ${ }^{1-8}$. La patogénesis de esta enfermedad se relaciona estrechamente con un reflujo gastroesofágico crónico y persistente ${ }^{9-11}$, compuesto principalmente por un reflujo mixto, tanto de contenido gástrico e intestinal, demostrado tanto experimentalmente ${ }^{12-14}$, como en numerosos estudios clínicos ${ }^{1-8,15}$. Su importancia clínica se relaciona al hecho que hay un significativo aumento del riesgo a desarrollar un adenocarcinoma, el que es 30 a 120 veces mayor comparado con la población general $^{5-7}$, con una incidencia de cáncer de $0,5 \mathrm{a} 1 \%$ por 100 pacientes-año. El adenocarcinoma esofágico es el cáncer que más ha aumentado en el mundo, con un incremento de $500 \%$ en los últimos 20 años, en especial en EEUU ${ }^{16}$. Nuestro grupo publicó por primera vez en Chile hace 30 años un estudio de 8 pacientes con adenocarcinoma avanzado de esófago, con nula sobrevida a 5 años ${ }^{17}$. El objetivo del presente estudio fue determinar las características clínicas y anatomo patológicas, así como la operación, la evolución postoperatoria y la sobrevida y los resultados a largo plazo en un grupo de pacientes con adenocarcinoma esofágico.

\section{Material y Método}

\section{Pacientes estudiados}

El presente análisis es de tipo descriptivo y retrospectivo, abarcando a los pacientes con adenocarcinoma esofágico y síndrome de Barrett extenso entre 1996 y 2011. Sólo se incluyeron a los pacientes sometidos a cirugía, excluyendo, por lo tanto, a 13 pacientes que presentaban metástasis a distancia con contraindicación de operabilidad, así como a pacientes con carcinoma escamoso del esófago o con adenocarcinoma del cardias. También se ex- cluyó a pacientes con esófago de Barrett corto que fueron incluidos en otra presentación ${ }^{18}$. Todos tenían un completo estudio endoscópico y biopsias, que confirmaban un adenocarcinoma esofágico y una tomografía axial computarizada que no demostraba metástasis a distancia. En 32 pacientes (60\%) también había un estudio radiológico contrastado con sulfato de bario.

\section{Definición}

Se consideró un adenocarcinoma de esófago aquel tumor que estaba a $5 \mathrm{~cm}$ proximal a la línea $\mathrm{Z}$ o cambio de mucosas, respetando por lo tanto, la mucosa del esófago distal y la unión gastroesofágica.

\section{Revisión de los resultados}

Los pacientes que se operaron entre 1996 y 2003 fueron revisados en forma retrospectiva. Sin embargo, desde 2004 se creó en el Departamento de Cirugía una unidad de registro y seguimiento prospectivo de todos los pacientes oncológicos, a cargo de una enfermera especializada en este tema (S.C). El análisis de su funcionamiento y sus resultados han sido publicados recientemente ${ }^{19}$. Este registro permite controlar a los pacientes cada 6 meses y conocer con exactitud la sobrevida de ellos.

\section{Cálculo estadístico}

Para el análisis de los resultados, se empleó el promedio con desviación estándar. La sobrevida actual se calculó de acuerdo a la fórmula de KaplanMeier.

\section{Resultados}

La Tabla 1 muestra el cambio epidemiológico en cuanto a su frecuencia relativa comparado con el total de cánceres esofágicos resecados (escamoso y adenocarcinoma) en los últimos 43 años en el Departamento de Cirugía.

Se aprecia un aumento de 10 veces su frecuencia relativa comparada con los resultados 30 a 40 años atrás. La Tabla 2 analiza las características clínicas de los 53 pacientes con adenocarcinoma esofágico 
Tabla 1. Frecuencia relativa del adenocarcinoma esofágico en el total de esofaguectomías por cáncer realizadas entre 1968 y 2011

\begin{tabular}{|cccc|}
\hline Período & $\begin{array}{c}\text { n total } \\
\text { esofaguectomías } \\
\text { por cáncer }\end{array}$ & n AdenoCa. & $\%$ \\
\hline $1968-1983$ & 137 & 7 & 5 \\
\hline $1984-1995$ & 108 & 16 & 15 \\
\hline $1996-2003$ & 60 & 22 & 36 \\
\hline $2004-2011$ & 62 & 31 & 50 \\
\hline
\end{tabular}

Tabla 2. Características clínicas de 53 pacientes con adenocarcinoma esofágico (1996-2011)

\begin{tabular}{|cll|}
\hline - Edad promedio (años) & $58,1 \pm 8,6(22-78)$ \\
- Género & Femenino & 8 \\
Masculino & $45(84,9 \%)$ \\
- T ${ }^{\circ}$ síntomas (meses) & $90(1-240)$ \\
Asintomáticos & $5(9,4 \%)$ \\
Síntomas crónicos & Pirosis $47(88,7 \%)$ \\
& Regurgitación $40(75,5 \%)$ \\
& Dínfagia $45(85 \%)$ \\
& Baja de peso 40 $(75,5 \%)$ \\
& Hemorragia digestiva $2(3,8 \%)$ \\
& Dolor epigástrico o retroesternal \\
& $15(28,3 \%)$ \\
& Más de 1 síntoma $38(71,7 \%)$ \\
&
\end{tabular}

Tabla 3. Tipo de operación realizada en 53 pacientes con adenocarcinoma esofágico

\begin{tabular}{|llc|}
\hline 1. Resecabilidad & $51(96,2 \%)$ & \\
NO resecables & $2(3,8 \%)$ metástasis peritoneales & \\
2. Tipo resección & Esofaguectomía total & $48(94,1 \%)$ \\
& Esófago-gastrectomía total & 3 \\
3. Abordaje & Transhiatal vídeo asistida & $36(70,6 \%)$ \\
& Transtorácica & $3(5,9 \%)$ \\
& Toracoscópica/laparoscópica & $12(23,5 \%)$ \\
4. Reemplazo & Estómago & $45(88,2 \%)$ \\
esofágico & Colon derecho & 6 \\
5. Ascenso & Retroesternal & $30(58,8 \%)$ \\
órgano & Mediastínico & 21 \\
6. Otras & Esplenectomía necesidad & 2 \\
operaciones & Colecistectomía & 15 \\
& Nefrectomía derecha & 1 \\
& Lesión bronquio fuente & 1 \\
& Piloroplastía & 1 \\
\hline
\end{tabular}

operados entre 1996 y el 2011. Hay un claro predominio del sexo masculino en una proporción de casi 6:1. La presencia de un esófago de Barrett extenso previo al diagnóstico de adenocarcinoma se conoció en el $53 \%$ de los pacientes, mientras que en 47\% no había antecedentes endoscópicos de este diagnóstico. De los 28 pacientes con Esófago de Barrett conocido, 6 de ellos habían sido operados en promedio 9 años antes de la aparición del tumor realizando una cirugía antireflujo clásica por reflujo gastroesofágico crónico patológico. Estos pacientes han sido publicados previamente $^{20,21}$. Los otros 22 pacientes habían recibido tratamiento con inhibidores de la bomba de protones en forma intermitente y con controles irregulares. Algunos de ellos han sido reportados previamente ${ }^{22}$. El tiempo de síntomas previos al diagnóstico de adenocarcinoma fue prolongado, pero sólo el 90\% de los casos presentaba síntomas crónicos o agudos. En un 10\% el adenocarcinoma fue un hallazgo endoscópico. Un gran porcentaje de pacientes presentaba más de 1 síntoma.

La Tabla 3 señala los detalles del procedimiento quirúrgico. Dos pacientes $(3,8 \%)$ no pudieron ser resecados por el hallazgo de metástasis peritoneales no detectadas en el estudio preoperatorio. La resección esofágica total se realizó en 48 pacientes, mientras que en 3 casos $(5,9 \%)$ se procedió a una esófago-gastrectomía total para extirpar los linfonodos de la región abdominal en forma completa. El abordaje se realizó de 3 maneras: a) transhiatal vídeo asistida especialmente antes del año 2006; b) transtorácica clásica con toracotomía en un bajo porcentaje y en los últimos años por vía mini-invasiva toracoscópica y/o laparoscópica en 23,5\% de los casos. El reemplazo del esófago se efectuó en la mayoría con el estómago y sólo en 12\% se utilizó el colon derecho. El ascenso del estómago o colon se realizó por vía retroesternal en casi $60 \%$ de los pacientes. Hubo algunas intervenciones agregadas que se detallan en la tabla.

La Tabla 4 describe la evolución postoperatoria de los 51 pacientes con resección esofágica. La morbilidad más importante fue la filtración de la anastomosis esófagogástrica o colónica a nivel cervical en un $55 \%$ de los pacientes, que fueron manejados con drenaje local, alimentación enteral a través de sonda nasoyeyunal y 
Tabla 4. Evolución postoperatoria de 51 pacientes con adenocarcinoma esofágico resecados

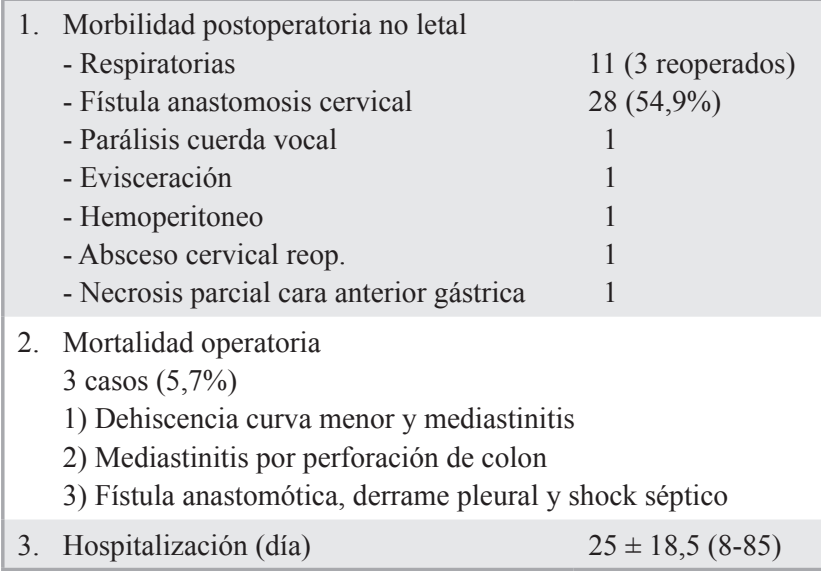

Tabla 5. Hallazgos histopatológicos del esófago resecado $\mathbf{n}=\mathbf{5 1}$

\begin{tabular}{|c|c|c|}
\hline - Largo metar & asia columnar & $7,4 \mathrm{~cm}(3-13)$ \\
\hline - Linfonodos & secados & $19 \pm 9(2-35)$ \\
\hline - Histología: & $\begin{array}{l}\text { Adenocarcinoma tubular o } \\
\text { tubulo papilar } 47\end{array}$ & $(92,1 \%)$ \\
\hline & $\begin{array}{l}\text { Adenocarcinoma mixto con } \\
\text { componentes mucinoso o en } \\
\text { anillo de sello }\end{array}$ & $4(7,9 \%)$ \\
\hline - Profundidad & nfiltración & \\
\hline & Mucosa-submucosa & 10 \\
\hline & Muscular & 12 \\
\hline & Transmural & 29 \\
\hline
\end{tabular}

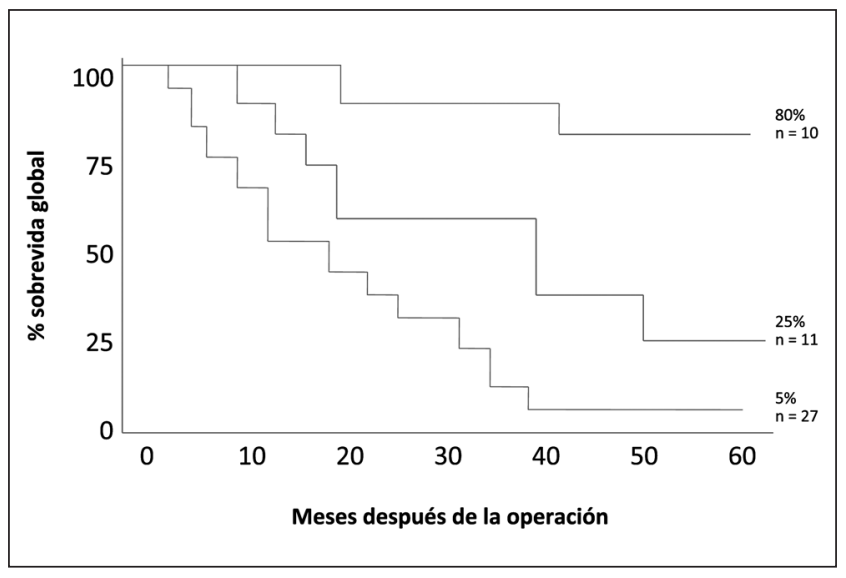

Figura 1 curaciones locales. Sólo 1 paciente necesitó una reoperación por un extenso absceso cervical. Complicaciones relacionadas con la esofaguectomía se presentaron como parálisis de la cuerda vocal, y necrosis parcial de la cara anterior del estómago en 1 caso cada uno. Hubo un quilotórax que requirió reoperación y drenaje.

La mortalidad operatoria corresponde a 3 pacientes $(5,7 \%), 1$ por dehiscencia curva menor del estómago ascendido y mediastinitis, una por mediastinitis por perforación de colon y un paciente con fístula anastomótica, derrame pleural y shock séptico.

La hospitalización postoperatoria promedio fue de 25 días, con rango entre 8 y 85 días. Los hallazgos histológicos se resumen en la Tabla 5. El largo promedio de la metaplasia columnar en el esófago fue de 7,4 $\mathrm{cm}$, teniendo todos los pacientes un esófago de Barrett extenso. Ningún paciente tenía el límite oral o caudal de la pieza quirúrgica resecada con infiltración tumoral (Lo y Lc negativas). El número de linfonodos resecados en promedio fue de 19 ganglios. Sólo 15 pacientes tenían linfonodos con ausencia de metástasis $(29,4 \%)$. Todos los casos correspondían a un adenocarcinoma tubular o tubulo-papilar, pero en 7,9\% el carcinoma fue mixto, teniendo también un componente mucinoso o un anillo de sello. Hubo 10 pacientes que presentaron un cáncer incipiente (mucoso o submucoso) 12 pacientes con un cáncer hasta la capa muscular y 29 pacientes con carcinoma que infiltraba en forma transmural.

La sobrevida actuarial de los 48 pacientes que no fallecieron en el período postoperatorio se muestra en la Figura 1. Se aprecia una diferencia muy significativa de la sobrevida a 5 años entre pacientes con cáncer incipiente, comparados con el cáncer muscular o transmural $(\mathrm{p}<0,01)$.

\section{Discusión}

Los resultados del presente estudio sugieren algunos hechos importantes de destacar: a) aumento del adenocarcinoma esofágico en la última década comparados a décadas anteriores; b) sólo un porcentaje de estos pacientes tienen el diagnóstico previo del esófago de Barrett; c) el cambio de abordaje quirúrgico desde la cirugía clásica con toracotomía hasta un abordaje mini-invasivo. 
El enorme aumento de la incidencia de adenocarcinoma esofágico ha sido reportado en varios estudios, destacando un $500 \%$ de aumento comparado con cifras de 30 a 40 años atrás ${ }^{4,5,16}$. En nuestro país no existen otras publicaciones referentes a este tema, salvo el nuestro de hace 30 años ${ }^{17}$. Sólo hay 2 reportes aislados de casos operados junto con carcinoma epidermoide. Uno es de Venturelli y $\operatorname{cols}^{23}$, que reportan 1 paciente vivo entre 5 esofaguectomías a más de 10 años de control. Otro es la referencia de Butte y $\operatorname{cols}^{24}$, quienes reportan que de 39 pacientes con adenocarcinoma de la unión gastroesofágica, 7 eran adenocarcinoma con esófago de Barrett. Sin embargo, también comprobamos un aumento significativo, desde 5\% en la década del 70 hasta un $50 \%$ en los últimos 7 años. Esto significa que los pacientes con síntomas crónicos de reflujo gastroesofágico y un esófago de Barrett extenso deben incluirse en un estricto protocolo de seguimiento endoscópico e histológico, para prevenir el desarrollo de adenocarcinoma como lo demostramos en un estudio nuestro ${ }^{22}$.

Otro importante aspecto en estos pacientes fue el hecho que en sólo un 50\% de los casos se conocía el diagnóstico previo de esófago de Barrett. En la literatura se describe que incluso sólo en 5\% de 1.503 casos $^{25}$ con adenocarcinoma esofágico tuvieron el diagnóstico previo de E.B. Otros autores señalan esta cifra en un $15 \%{ }^{2}$.

Nuestras cifras de morbilidad y mortalidad operatoria $(5 \%)$ están de acuerdo a lo publicado por otros autores ${ }^{20,27}$. El porcentaje de linfonodos positivos en adenocarcinoma esofágico es mayor que en los adenocarcinomas gástricos. En presencia de un carcinoma submucoso, esta cifra se ha reportado en un $22 \%{ }^{28}$, lo que indica la necesidad de un muy completo estudio preoperatorio en la presencia de cáncer incipiente, incluyendo la endosonografia para precisar el correcto diagnóstico referente a la profundidad de la infiltración tumoral en la pared del esófago.

Últimamente ha habido varios avances en el diagnóstico y tratamiento de este tumor tan prevalente ${ }^{29}$ :

a. Diagnóstico y tratamiento endoscópico.

b. Nuevas técnicas quirúrgicas, con el uso del abordaje mini-invasivo.

c. El empleo de medicamentos que frenan la aparición de Adenocarcinoma.

d. Nuevas estrategias de tratamiento neo-adyuvante.

e. Terapias moleculares en investigación y evaluación.

El diagnóstico endoscópico se ha ido perfeccionando con nuevos equipos altamente sofisticados. Pero el gran avance ha sido la cirugía endoscópica, ya sea ablativa o con resección de la mucosa, que ha tenido avances notables. Hay múltiples estudios recientes ${ }^{30-32}$ que han demostrado básicamente lo siguiente:

1. Que el tratamiento endoscópico es una alternativa efectiva en cuanto a morbilidad y mortalidad a la esofaguectomía en pacientes con displasia de alto grado y adenocarcinoma incipiente ${ }^{30,31}$.

2. Que se requiere de múltiples procedimientos para lograr su objetivo ${ }^{31}$.

3. Sin embargo, la recurrencia del cáncer es mayor con la resección endoscópica, por lo que aún es mandatorio un seguimiento alejado para demostrar su real efectividad ${ }^{30}$.

En cuanto a nuevas técnicas quirúrgicas, el abordaje mini-invasivo ha sido ampliamente desarrollado por numerosos autores, con muy buenos resultados, similares a la esofaguectomía estándar ${ }^{27,32-35}$. Los resultados publicados sugieren que:

a. La cirugía mini-invasiva es segura, efectiva, con menor morbimortalidad comparados con la esofaguectomía estándar ${ }^{27,33,34}$.

b. La cirugía mini-invasiva es una alternativa válida con resultados oncológicos similares a la esofaguectomía estándar ${ }^{33,34}$.

c. Sin embargo, un estudio poblacional en Inglaterra demostró que aunque es un abordaje seguro, no tenía mayores beneficios en morbilidad y mortalidad operatoria e incluso se acompañaba de más re-intervenciones ${ }^{27}$.

En nuestro grupo, 2 cirujanos (IB y GC) han desarrollado este tipo de abordaje toracoscópico y laparoscópico, con anastomosis siempre en el cuello ${ }^{36}$.

Sin embargo, un autor con gran experiencia ${ }^{34}$, comparando en 1.000 pacientes con abordaje de McKeown con el de Ivor Lewis (anastomosis intratoraccica) ha preferido este último procedimiento. Es interesante señalar que se ha demostrado que el empleo de inhibidores de la bomba de protones, antiinflamatorios, aspirinas y estatinas ${ }^{37}$, disminuye o frenan la aparición de adenocarcinoma, pero faltan aún más estudios para probar esta hipótesis.

En resumen, el adenocarcinoma esofágico en esófago de Barrett ha aumentado su prevalencia 10 veces comparado con 40 años atrás. Es imprescindible el seguimiento endoscópico e histológico de pacientes con esófago de Barrett. El abordaje quirúrgico puede ser por vía transhiatal video asistida o por abordaje mini-invasivo. La sobrevida a 5 años es similar a la reportada por nuestro grupo en un estudio previo $^{38}$.

\section{Referencias}

1. Spechler SJ, Goyal RK. Barrett's esophagus. New Engl J Med. 1986;315:362-71. 
2. Cameron AJ, Lomboy CT. Barrett's esophagus: age, prevalence and extent of columnar epithelium. Gastroenterology 1992;103:1241-5.

3. Spechler $\mathrm{Sj}$, Goyal RK. The columnar lined esophagus, intestinal metaplasia and Norman Barrett. Gastroenterology 1996;110:614-21.

4. Spechler SJ. The columnar lined esophagus. Gastroent Clin N Am. 1997;26:455-66.

5. DeMeester TR, Peters JH, DeMeester TR. Barrett's esophagus. Curr Probl Surg. 2001;38:549-640.

6. Falk GW. Barrett's esophagus. Gastroenterology 2002;122:1569-79.

7. Csendes A, Smok G, Quiroz J, Burdiles P, Rojas J, Castro $\mathrm{C}$, et al. Clinical, endoscopic and functional studies in 408 patients with Barrett's esophagus compared to 174 cases with intestinal metaplasia of the cardia. Am J Gastroent. 2002;97:574-60.

8. Csendes A. Results of antireflux surgery in patients with Barrett's esophagus. Europ Surg. 2008;4:154-64.

9. Lagergen J, Bergstrom R, Lindberg A, Nyren O. Symptomatic gastroesophageal reflux as a risk factor for esophageal adenocarcinoma. New Eng J Med. 1999;340:825-30.

10. Solaymani-Dodaran M, Logan RIA, West J, Card T, Coupland C. Risk of oesophageal cancer in Barrett's oesophagus and gastro-oesophageal reflux. Gut 2004;53:1070-4.

11. Eisen GM, Sandler RS, Murray S, Gottfried M. The relationship between gastroesophageal reflux disease and its complications with Barrett's esophagus. Am J Gastroent. 1997;92:27-31.

12. Attwood SEA, Smyrk TC, DeMeester TR, Mirvish SS, Stein HJ, Hinder RA. Duodenoesophageal reflux and the development of esophageal carcinoma in rats. Surgery 1992;111:503-10.

13. Fein M, Peter JH, Chandrasoma P, Ireland AP, Oberg $\mathrm{S}$, Ritter MP, et al. Duodenoesophageal reflux induces esophageal adenocarcinoma without exogenous carcinogen. J Gastrointest Surg. 1998;2:260-8.

14. Nishijima K, Miwa K, Miyoshita T, Kirani S, Ninomiya I, Fushida $\mathrm{S}$, et al. Impact of the biliary diversion procedure on carcinogenesis in Barrett's esophagus surgically induced by duodenoesophageal reflux in rats. Ann Surg. 2004;240:57-67.

15. Sarela AJ, Hick DG, Verbeke CS, Casey JF, Guillou PJ, Clark GNB. Persistent acid and bile reflux in asymptomatic patients with Barrett's esophagus receiving proton pump inhibitor therapy. Arch Surg. 2004;139:547-51.

16. Bodchand V, Sampliner RE. Risk for cancer in Barrett's esophagus: Medical versus surgical therapy. Curr Gastroent Report 2007;9:189-94.

17. Csendes A, Ramírez C, Smok G, Braghetto I, Velasco N. Adenocarcinoma primario del esófago en pacientes con estenosis esofágica y síndrome de Barrett. Rev Méd Chile 1983;111:1255-8.

18. Cortés S, Csendes A, Yarmuch J. Resultados de la unidad de oncología del Departamento de Cirugía de un hospital universitario (2004-2010). Rev Chil Cir. 2011;63:534-7.

19. Csendes A, Braghetto I, Burdiles P, Smok G. Adenocarcinoma developing in short-segment Barrett's esophagus: analysis of 5 patients and review of the literature. Europ Surg. 2007;39:359-63.

20. Csendes A, Burdiles P, Braghetto I, Smok G, Castro C, Korn O, et al. Dysplasia and adenocarcinoma after classic antireflux surgery in patients with Barrett's esophagus: the need for long term subjective and objective follow up. Ann Surg. 2002;235:178-85.

21. Csendes A, Burdiles P, Braghetto I, Korn O. Adenocarcinoma appearing very late after antireflux surgery for Barrett's esophagus: long term follow up review of the literature and addition of 6 patients. J Gastrointest Surg. 2004;8:434-41.

22. Burdiles P, Csendes A, Smok G, Braghetto I, Korn O. Progresión de metaplasia intestinal o Adenocarcinoma en esófago de Barrett: utilidad de la vigilancia endoscópica. Rev Med Chile 2003;131:587-96.

23. Venturelli A, Sánchez A, Cardimil B, Díaz J, Jimenes J, Carcamo C. Cáncer de esófago. Sobrevida a 10 años plazo. Rev Chil Cir. 2001;53:541-4.

24. Butte JM, Becker F, Visscher A, Waugh E, Meneses M, Court I, et al. Cáncer de la unión gastroesofágica. Evaluación de los resultados quirúrgicos, sobrevida alejada y factores pronósticos en enfermos con terapia resectiva. Rev Med Chile 2010;138:53-60.

25. Gorhbein J, Weinstein NM. Preoperative prevalence of Barrett's esophagus in Esophageal adenocarcinoma: a systematic review. Gastroenterology 202;122:26-34.

26. Owrebo K, Lie SA, Laerum OD, Soanes K. Long term survival of adenocarcinoma of the esophagus after transthoracic and transhiatal esophagectomy. World J Surg Oncol. 2012;10:130-6.

27. Mamidanna R, Bottle A, Aylin P, Faiz O, Hanna GB. Short term outcomes following open versus minimally invasive esophagectomy for cancer in England. A population based national study. Ann Surg. 2012;255:197203.

28. Leers JM, DeMeester SR, Oezcelik A, Klipfel N, Ayazi S. The prevalence of lymphnode metastasis patients with T1 esophageal carcinoma. Ann Surg. 2011; 253:271-8

29. Behrens A, Pech O, Groupe F, May A, Lorenz D, Ell C. Barrett's adenocarcinoma of the esophagus: better outcomes through new methods of diagnosis and treatment. Dtsch Arztebl Int. 2012;109:48.

30. Menon D, Srafino KT, Wu Hi copic treatments for Barrett's esophagus: a systematic review of safety and effectiveness compared to esophagectomy. BMC Gastroent. 2010;27:10:111.

31. Zehetner J, DeMeester SR, Hagen JA, Ayazi S. Endoscopic resection and ablation versus esophagectomy for high grade dysplasia and intramucosal carcinoma. J 
Thorac Cardiovasc Surg. 2011;141:39-47.

32. Pech O, Bollschweiler E, Manner H, Leers G, Ell Ch, Hölscher AH. Comparison between endoscopic and surgical resection of mucosal esophageal adenocarcinoma in Barrett's esophagus at two high-volume centers. Ann Surg. 2011;254:67-72.

33. Dantoc C, Cox MR, Eslick GD. Evidence to support the use of minimally invasive esophagectomy for esophageal cancer: A meta analysis. Arch Surg. 2012;147:76876.

34. Inketich JD, Pennathur A, Awais O, Levy RM. Outcomes after minimally invasive esophagectomy: review over 1000 patients. Ann Surg. 2012;256:95-103.

35. Dantoc MM, Cox MR, Eslick GD. Does minimally invasive esophagectomy provide comparable oncologic outcome to open techniques? A systematic review. J Gastrointest Surg. 2012;16:486-96.

36. Braghetto I, Csendes A, Cardemil G, Burdiles P, Korn $\mathrm{O}$, Valladares $\mathrm{H}$. Open transthoracic or transhiatal esophagectomy versus minimally invasive esophagectomy in terms of morbidity, mortality and survival. Surg Endosc. 2006;20:1681-6.

37. Nguyen DM, Richardson P, El-Serag HB. Medications (NSAID, Status, PPI) and the risk of esophageal adenocarcinoma in patients with Barrett's esophagus. Gastroenterology 2010;138:2260-8.

38. Braghetto I, Csendes A, Cornejo A, Amat J, Cardemil G, Burdiles P, et al. Sobrevida de pacientes con cáncer de esófago sometidos a Esofaguectomía total torácica. Rev Med Chile 2000;128:64-74. 